\title{
Graph Visualization
}

\author{
Jarke J. van Wijk \\ Eindhoven University of Technology, Eindhoven, The Netherlands \\ vanwijk@win.tue.nl \\ http://www.win.tue.nl/ vanwijk
}

Black and white node link diagrams are the classic method to depict graphs, but these often fall short to give insight in large graphs or when attributes of nodes and edges play an important role. Graph visualization aims obtaining insight in such graphs using interactive graphical representations. A variety of ingredients, including color, shape, 3D, shading, and interaction can be used to this end. In this invited talk an overview is given of work on graph visualization of the visualization group of Eindhoven University of Technology, The Netherlands. A wide variety of examples is shown and discussed using demos and animations.

One focus of the group has been software visualization, aiming towards the development of technology that makes it easier to understand the structure of large software artifacts. An early example were cushion treemaps, developed to visualize hierarchical data, in particular file systems (SequoiaView), another more frivolous example are our botanically inspired tree visualizations. State space models lead to very large, but also often symmetrical graphs, which can be exploited to obtain clear and compact visualizations. Combinations of hierarchical data and networks occur often in practice, a typical case is the visualization of call-graphs of software systems. Such data can be shown using an interactive incidence-matrix or using hierarchical edge bundles.

Besides the development of new methods and techniques, evaluation is an important and often difficult aspect. Details of visualizations can be evaluated using controlled user experiments, examples are the assessment of proper scales for icons and different ways to show edge direction.

Finally, some other work related to graphs is shown. Myriahedral projections are a new method to generate cartographic maps almost without distortion that lean heavily on graphs. Platonic solids, such as the cube and dodecahedron, are examples of so-called regular maps: highly symmetric graphs, embedded on a surface. Examples of such regular maps for surfaces of genus 2 and higher are presented. 